Article

\title{
Cooperative Game-Based Synergistic Gains Allocation Methods for Wind-Solar-Hydro Hybrid Generation System with Cascade Hydropower
}

\author{
Liqin Zhang ${ }^{1}$, Jun XIE ${ }^{1, *(\mathbb{D})}$, Xingying CHEN ${ }^{1}$, Yongsheng Zhan ${ }^{2}$ and Lv Zhou ${ }^{2}$ \\ 1 College of Energy and Electrical Engineering, Hohai University, Nanjing 211100, China; \\ lqzhang@hhu.edu.cn (L.Z.); xychen@hhu.edu.cn (X.C.) \\ 2 Yalong River Hydropower Development Company LTD, Chengdu 610051, China; \\ zhanyongsheng@ylhdc.com.cn (Y.Z.); zhoulv@ylhdc.com.cn (L.Z.) \\ * Correspondence: eejxie@163.com; Tel.: +86-25-5714-3197
}

Received: 4 July 2020; Accepted: 29 July 2020; Published: 30 July 2020

\begin{abstract}
In order to encourage hybrid generation of multiple wind/solar/hydro power stakeholders, synergistic gains from hybrid generation should be allocated fairly, efficiently and reasonably to all power stakeholders. This paper explores how cooperative game theory resolves conflicts among multiple wind/solar/hydro power stakeholders. Elaborate allocation processes of the nucleolus, Shapley value and MCRS methods are presented in resolve synergistic gains allocation problems of wind-solar-hydro hybrid generation system with cascade hydropower. By analyzing properties such as existence, uniqueness and rationality, we find that both the Shapley value and MCRS methods are fair, efficient and rational allocation methods whereas the nucleolus method is limited by reservoir volume of hydro power stakeholders. Analyses on computational feasibility show that the Shapley value method may induce combinational explosion problem with the integration of more power stakeholders. A further application in Yalong River basin demonstrates that, compared with the Shapley value method, the MCRS method significantly simplifies allocating process and improves computational efficiency. Therefore, the MCRS method is recommend as a fair, efficient, rational and computational feasible allocation method for hybrid generation system with large number of wind/solar/hydro power stakeholders.
\end{abstract}

Keywords: wind-solar-hydro hybrid generation; synergistic gains allocation; nucleolus method; Shapley value method; MCRS method

\section{Introduction}

With raising concerns about environmental pollution, the need for renewable energies are increasing dramatically. Renewable energy generation has already been developed as one of the mainstream generation approaches over the past years [1,2]. According to a global renewables report, the capacity of renewable energy generation has increased by $181 \mathrm{GW}$ in 2018 and the production of renewable energy generation account for approximately $26 \%$ of global electricity generation by the end of 2018 [3].

As a flourishing renewable energy generation technology, hybrid generation of wind, solar and hydro power shows great superiority in regulating and utilizing natural resources with lower economic-environmental costs [4-6]. In order to develop long-term and mutual-beneficial partnerships, all power stakeholders should be brought together in a new way of communication, cooperation and decision-making [7]. Many recent studies have focused on the allocation of generation resources, such as firm energy rights allocation of hydro power stakeholders [8,9], optimal storage allocation of multiple 
reservoirs stakeholders [10], wind-solar capacity allocation [11] and solar radiation allocation [12]. However, the absence of synergistic gains allocation methods could collapse the cooperation of multiple power stakeholders. For example, if the reduced generation of those generation-reducing power stakeholders could not be compensated and the increased generation of those generation-increasing power stakeholders could not be shared, some power stakeholders would prefer independent generation rather than hybrid generation. $\mathrm{Xu}$ et al. [13] proposed a synergistic revenue allocation method based on the Nash-Harsanyi bargaining model (NHBM) and simulated allocation results of a cascade of four hydro power stakeholders. However, the NHBM is a static model which could not describe uncertainty and probabilistic correlation of wind and solar energy. proportional method (PM), marginal benefits method (MBM) and last addition method (LAM) are also applied to allocate synergistic gains of a wind-solar-hydro hybrid generation system with cascade hydropower [14]. The PM allocates synergistic gains proportionately according to the installed generation capacity, which neglects the volatility and uncertainty of wind and solar resources. The MBM decides the allocations of all power stakeholders according to their marginal contributions to grand coalition, which may become unfair for power stakeholders who sacrifice their own generation for cooperative generation, e.g., upstream hydro power stakeholders. The LAM allocates synergistic gains in proportion to the additional generation that each power stakeholder brings to grand coalition, which may not suitable for power stakeholders with particularly large gaps in generation ability.

This paper applies cooperative game-based methods in allocating synergistic gains of a hybrid generation system with multiple wind/solar/hydro power stakeholders. Cooperative game theory provides an effective way to deal with interactive conflicts among multiple stakeholders and allocate cooperative gains under binding agreements [15]. As classic cooperative game-based allocation methods, the nucleolus and Shapley value methods have been widely applied in resolving allocation problems such as multiple wind power producers [16], wind-solar [17] wind-hydro [18,19] and cascade hydro [8] generation systems. Baeyens et al. [16] applied nucleolus theory to identify fair sharing mechanism among independent wind power producers. Tzavellas et al. [17] modelled cooperation between several virtual synchronous generator units with large number of wind turbines and PV arrays using the nucleolus method. Alexandre et al. [18] proposed core-based methods (e.g., nucleolus method and proportional nucleolus method) to allocate financial gains of hybrid wind-hydro generation system in renewable energy hedge pool. Zima-Bockarjova et al. [19] proposed Shapley value-based collaboration scheme to share synergistic profits from coordinated operation strategy of a wind-hydro generation system. Faria et al. [8] investigated the firm energy rights of hydro plants which impose maximum contract limit in electricity market and applied the Shapley value method in the firm energy rights allocation problem.

However, the nucleolus method and the Shapley value method will bring about enormous computational burden. In order to simplify allocation process, Heaney et al. [20] investigated boundaries of the core and proposed a novel allocation method, named minimum cost-remaining savings (MCRS) method. In [20], the MCRS method was first applied to allocate costs of a multi-hydro resource project and proved to be fair. In this paper, we further investigate the potentials of MCRS method in allocating synergistic gains of a wind-solar-hydro hybrid generation system with cascade hydropower.

The main contributions of this paper are as follows:

(1) Elaborate allocation processes of the nucleolus, Shapley value and MCRS methods are presented to allocate synergistic gains of a wind-solar-hydro hybrid generation system with cascade hydropower;

(2) Both the Shapley value and MCRS methods are recommended as fair, efficient and rational synergistic gains allocation methods for hybrid generation system with multiple wind/solar/hydro power stakeholders. The limitation of nucleolus method is also presented;

(3) The MCRS method is much practical and computational feasible for synergistic gains allocation problems with large number of wind/solar/hydro power stakeholders, compared with the Shapley value method. 
Three major procedures of this study are depicted in Figure 1 and the details are provided in the following sections.

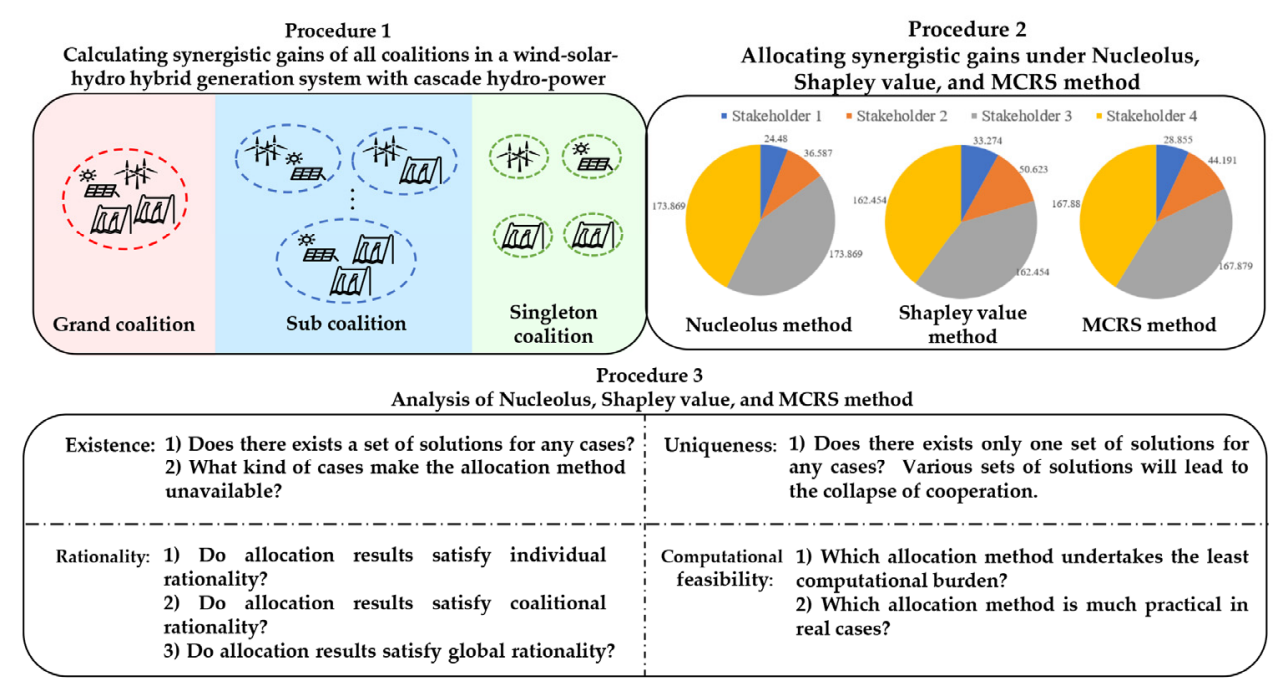

Figure 1. Three major procedures of this paper.

\section{Synergistic Gains of Wind/Solar/Hydro Hybrid Generation Coalitions}

Synergistic gain of a wind/solar/hydro hybrid generation coalition can be quantified as the additional generation of joint optimal operation, compared with individual optimal operation. In joint optimal operation model, all power stakeholders are unified regulated to achieve maximum generation of the coalition. In individual optimal operation model, generation of a coalition is the sum of maximum generation of all power stakeholders. Hence, the synergistic gain of a hybrid generation coalition $S$ can be quantified as:

$$
\begin{aligned}
\Delta P_{S} & =P_{S}^{(1)}-P_{S}^{(0)}=P_{S}^{(1)}-\sum_{i \in S} P_{i}^{(0)} \\
& =v(S)-\sum_{i \in S} v(i)
\end{aligned}
$$

where, $\Delta P_{S}$ is the synergistic gain of hybrid generation coalition $S ; P_{S}^{(1)}$ and $P_{S}^{(0)}$ are the generation under joint and individual optimal operation model, respectively; $\sum_{i \in S} P_{i}^{(0)}$ is the total generation of $|S|$ power stakeholders in coalition $S$ under individual optimal operation models. Assuming $v(\cdot)$ as the characteristic function, $v(S)$ is the generation of hybrid generation coalition $S$ under joint optimal operation model and $v(i)$ is the generation of power stakeholder $i$ under individual optimal operation model.

Generation of all power stakeholders and all possible hybrid generation coalitions are determined by solving joint optimal operation models and individual optimal operation models of a wind-solar-hydro hybrid generation system with cascade hydropower [14].

\section{Cooperative Game-Based Synergistic Gains Allocation Methods}

Allocating synergistic gains of a wind-solar-hydro hybrid generation system requires to analyze the interactive conflicts and competing claims of all power stakeholders. A fair, efficient and rational allocation method will be of great significance to develop partnerships of all power stakeholders. Here, elaborate allocation process of nucleolus, Shapley value and MCRS method are presented in resolving synergistic gains allocation problems of wind-solar-hydro hybrid generation system. 


\subsection{Nucleolus Method}

The theoretical basis of nucleolus method is to minimize the degree of dissatisfaction of the most unsatisfactory coalition. For synergistic gains allocation problems, the dissatisfaction of a hybrid generation coalition comes from the excess value of synergistic gain from hybrid generation compared with the sum of allocated synergistic gains. Hence, the practical basis of nucleolus method is to minimize the maximum excess value of all possible hybrid generation coalitions as:

$$
\begin{gathered}
e\left(S, y^{N u}\right)=\Delta P_{S}-\sum_{i \in S} y_{i}^{N u} \\
\left\{\begin{array}{l}
\varphi\left(y^{N u}\right)=\max _{S \subseteq N}\left(S, y^{N u}\right) \\
\varphi\left(y^{N u}\right) \leq \varepsilon
\end{array}\right.
\end{gathered}
$$

where, $y_{i}^{N u}$ is the allocated synergistic gains of power stakeholder $i$ under nucleolus method; $N$ is grand coalition composed of all power stakeholders; $e\left(S, y^{N u}\right)$ is the excess value of hybrid generation coalition $S(S \subseteq N) ; \varphi\left(y^{N u}\right)$ is the maximum excess value of all possible hybrid generation coalitions; $\varepsilon$ is an arbitrary small number.

The minimization problem Equation (3) can be incorporated into a linear programming problem as:

$$
\begin{aligned}
& \text { Min } \varepsilon \\
& \text { s.t. } \Delta P_{N}=\sum_{i \in N} y_{i}^{N u} \\
& \qquad \Delta P_{S}-\sum_{i \in S} y_{i}^{N u} \leq \varepsilon(S \subseteq N)
\end{aligned}
$$

where, the equality constraint ensures synergistic gains of grand coalition $N$ could be totally allocated to all power stakeholders; and the inequality constraint minimizes the excess value of all hybrid generation coalitions.

Thus, allocation result can be defined as the summation of allocated synergistic gain and the generation from individual operation as:

$$
x_{i}^{N u}=y_{i}^{N u}+v(i)
$$

where, $x_{i}^{N u}$ is allocation result of power stakeholder $i$ under nucleolus method.

\subsection{Shapley Value Method}

The basic idea of the Shapley value is to allocate synergistic gains in accordance with the marginal contribution of all power stakeholders. However, the marginal contribution that a power stakeholder brings to a possible hybrid generation coalition is greatly influenced by the entrance order. The Shapley value defines a weighted marginal contribution as:

$$
\begin{gathered}
\Delta P_{\hat{S}}=v(\hat{S})-\sum_{r \in \hat{S}} v(r) \\
W(\hat{S})=\frac{(|N|-|\hat{S}|) !(|\hat{S}|-1) !}{|N| !}\left(\Delta P_{\hat{S}}-\Delta P_{\hat{S}-\{i\}}\right)
\end{gathered}
$$

where, $\hat{S}$ is a hybrid generation coalition that power stakeholder $i$ participates; respectively; $\Delta P_{\hat{S}}-\Delta P_{\hat{S}-\{i\}}$ is the marginal contribution that power stakeholder $i$ makes to $\hat{S} ;(|N|-|\hat{S}|) !$ refers to all possible entrance orders that power stakeholder $i$ participates $\hat{S}$ at the first place; $(|\hat{S}|-1)$ ! refers to all possible entrance orders that power stakeholder $i$ participates $\hat{S}$ at the last place; $W(\hat{S})$ describes the weighted marginal contribution that power stakeholder $i$ brings to $|\hat{S}|$-stakeholder coalitions. Hence, 
Equation (7) guarantees that all power stakeholders can be treated fairly in all hybrid generation coalitions containing themselves.

Thus, allocated results and allocation result are:

$$
\begin{aligned}
& y_{i}^{S p}=\sum_{\hat{S} \subseteq N} W(\hat{S}) \\
& x_{i}^{S p}=y_{i}^{S p}+v(i)
\end{aligned}
$$

where, $y_{i}^{S p}$ and $x_{i}^{S p}$ are allocated synergistic gain and allocation result of power stakeholder $i$ under Shapley value method.

\subsection{MCRS Method}

The MCRS method delineates the upper and lower boundaries of the core by integrating information of intermediate hybrid generation coalitions. MCRS method includes three steps as: (1) calculate the maximin and minimum allocations of all power stakeholders; (2) calculate the sharing proportion of all power stakeholders; (3) allocate synergistic gains according to sharing proportion.

In step (1), the upper and lower boundaries of allocated synergistic gains of all power stakeholders can be obtained by solving a linear programming problem as:

$$
\begin{aligned}
& \text { Max or Min } y_{i}^{M C} \\
& \text { s.t. } \sum_{i \in N} y_{i}^{M C}=\Delta P_{N} \\
& \quad \sum_{r \in\{N-\{i\}\}} y_{r}^{M C} \leq \Delta P_{N}-\Delta P_{N-\{i\}}
\end{aligned}
$$

where, $y_{i}^{M C}$ is the allocated synergistic gain of power stakeholder $i$ under MCRS method. As can be seen from (10) that the equality constraint indicates the synergistic gain of grand coalition $N$ should be totally allocated to all power stakeholders and inequality constraint indicates that the sum of allocated synergistic gains of remaining stakeholders is no more than the synergistic gain when power stakeholder $i$ exits grand coalition $N$.

According to the complementarities of all wind/solar/hydro power stakeholders, the maximum and minimum allocations can also be regarded as:

$$
\begin{gathered}
y_{i, \max }=\Delta P_{N}-\Delta P_{N-\{i\}} \\
y_{i, \text { min }}=\Delta P_{\{i\}}
\end{gathered}
$$

where, maximum allocation $y_{i, \max }$ is regarded as the marginal contribution that power stakeholder $i$ makes to the synergistic gain of grand coalition $N$; and minimum allocation $y_{i, \min }$ is regarded as the synergistic gain of power stakeholder $i$ under individual optimal operation model.

In step (2), the sharing proportion is defined according to the differences between maximum and minimum allocations as:

$$
\gamma_{i}=\frac{y_{i, \max }-y_{i, \min }}{\sum_{r \in N}\left(y_{r, \max }-y_{r, \min }\right)}
$$

where, $\gamma_{i}$ is the sharing proportion of power stakeholder $i$.

In step (3), the allocated synergistic gain of each power stakeholders is:

$$
y_{i}^{M C}=y_{i, \min }+\gamma_{i} \Delta P_{N}
$$

Hence, allocation result under the MCRS method is:

$$
x_{i}^{M C}=y_{i}^{M C}+v(i)
$$




\section{Case Studies}

Synergistic gains from several wind-solar-hydro hybrid generation cases are allocated under the nucleolus, Shapley value and MCRS methods in this section. All cases are studied on a PC with $1.60 \mathrm{GHz}$ processors and 8.0 GB of RAM. CPLEX 12.4 is used to quantify generation from optimal wind/solar/hydro operation models while MATLAB 2017b is used to resolve synergistic gains allocation problems.

\subsection{Synergistic Gains Allocation of Four Power Stakeholder Hybrid Generation System}

In this case, there are one wind power stakeholder, one solar power stakeholder and a cascade of two hydro power stakeholders (namely power stakeholder 1, 2, 3 and 4). The installed capacity (IC) of power stakeholder 1 and 2 are $100 \mathrm{MW}$ and $50 \mathrm{MW}$, respectively. Optimal operations of wind/solar power stakeholders are based on prediction intervals in Figures A1 and A2; and optimal operations of hydro power stakeholders are based on natural inflow volume in Figure A3. The maximum spinning reserve coefficient is set as $5 \%$ and the additional spinning reserve coefficient is also set as $5 \%$.

In total, there are $15\left(2^{4}-1\right)$ possible coalitions of a hybrid generation system with four wind/solar/hydro power stakeholders. Hence, the synergistic gains allocation problem of this hybrid generation system can be regarded as a 4-person cooperative game with 15 non-empty possible coalitions. The generation of all hybrid generation coalitions are listed in Table 1.

Table 1. Generation of all possible non-empty coalitions (MWh).

\begin{tabular}{cccc}
\hline Coalition & Generation & Coalition & Generation \\
\hline$\{1\}$ & 1100 & $\{2,4\}$ & 2205.148 \\
$\{2\}$ & 205.5 & $\{3,4\}$ & 4765.004 \\
$\{3\}$ & 2555.48 & $\{1,2,3\}$ & 3984.925 \\
$\{4\}$ & 1924.664 & $\{1,2,4\}$ & 3354.109 \\
$\{1,2\}$ & 1313.1 & $\{1,3,4\}$ & 5913.965 \\
$\{1,3\}$ & 3704.441 & $\{2,3,4\}$ & 5045.488 \\
$\{1,4\}$ & 3073.625 & $\{1,2,3,4\}$ & 6194.449 \\
$\{2,3\}$ & 2835.964 & & \\
\hline
\end{tabular}

Next, cooperative game-based methods (nucleolus, Shapley value and MCRS method) will be applied to allocating synergistic gains of the wind-solar-hydro hybrid generation system. Moreover, properties of the presented allocation methods will be compared in terms of existence, uniqueness, rationality and computational feasibility.

\subsubsection{Nucleolus Method Allocation Process}

According to quantification method Equation (1), synergistic gains of all power stakeholders under individual operation model are:

$$
\begin{gathered}
\Delta P_{\{1\}}=v(\{1\})-v(\{1\})=0, \\
\Delta P_{\{2\}}=0, \Delta P_{\{3\}}=0, \Delta P_{\{4\}}=0 .
\end{gathered}
$$

Synergistic gains of all two-stakeholder coalitions are:

$$
\begin{aligned}
& \Delta P_{\{1,2\}}=v(\{1,2\})-v(\{1\})-v(\{2\})=7.6, \\
& \Delta P_{\{1,3\}}=48.961, \Delta P_{\{1,4\}}=48.961, \Delta P_{\{2,3\}}=74.984, \\
& \Delta P_{\{2,4\}}=74.984, \Delta P_{\{3,4\}}=284.86 .
\end{aligned}
$$


Synergistic gains of all three-stakeholder coalitions are:

$$
\begin{aligned}
& \Delta P_{\{1,2,3\}}=v(\{1,2,3\})-v(\{1\})-v(\{2\})-v(\{3\})=123.945, \\
& \Delta P_{\{1,2,4\}}=123.945, \Delta P_{\{1,3,4\}}=333.821, \Delta P_{\{2,3,4\}}=359.844 .
\end{aligned}
$$

Synergistic gain of grand coalition is:

$$
\Delta P_{\{1,2,3,4\}}=v(\{1,2,3,4\})-v(\{1\})-v(\{2\})-v(\{3\})-v(\{4\})=408.805
$$

Then, linear programming model Equation (4) can be written as:

$$
\begin{aligned}
& \text { Min } \varepsilon \\
\text { s.t. } & y_{1}^{N u}+y_{2}^{N u}+y_{3}^{N u}+y_{4}^{N u}=408.805 \\
\varepsilon & \geq-y_{1}^{N u} \\
\varepsilon & \geq-y_{2}^{N u} \\
\varepsilon & \geq-y_{3}^{N u} \\
\varepsilon & \geq-y_{4}^{N u} \\
\varepsilon & \geq 7.6-y_{1}^{N u}-y_{2}^{N u} \\
& \geq \geq 48.961-y_{1}^{N u}-y_{3}^{N u} \\
& \varepsilon \geq 48.961-y_{1}^{N u}-y_{4}^{N u} \\
& \geq 74.984-y_{2}^{N u}-y_{3}^{N u} \\
\varepsilon & \geq 74.984-y_{2}^{N u}-y_{4}^{N u} \\
& \geq 284.86-y_{3}^{N u}-y_{4}^{N u} \\
& \varepsilon \geq 123.945-y_{1}^{N u}-y_{2}^{N u}-y_{3}^{N u} \\
& \varepsilon \geq 123.945-y_{1}^{N u}-y_{2}^{N u}-y_{4}^{N u} \\
& \geq 333.821-y_{1}^{N u}-y_{3}^{N u}-y_{4}^{N u} \\
& \geq \geq 359.844-y_{2}^{N u}-y_{3}^{N u}-y_{4}^{N u}
\end{aligned}
$$

Optimal solutions of the above problem are:

$$
y_{1}^{N u}=24.48, y_{2}^{N u}=36.587, y_{3}^{N u}=173.869, y_{4}^{N u}=173.869
$$

Therefore, the allocation results under nucleolus method are:

$$
x_{1}^{N u}=1124.48, x_{2}^{N u}=242.087, x_{3}^{N u}=2729.349, x_{4}^{N u}=2098.533 .
$$

4.1.2. Shapley Value Method Allocation Process

According to Equations (6)-(8), the allocated synergistic gains of all power stakeholders are:

$$
\begin{aligned}
y_{1}^{S p} & =\frac{3 ! \times 0 !}{4 !}\left(\Delta P_{\{1\}}-\Delta P_{\varnothing}\right) \\
& +\frac{2 ! \times 1 !}{4 !}\left[\left(\Delta P_{\{1,2\}}-\Delta P_{\{2\}}\right)+\left(\Delta P_{\{1,3\}}-\Delta P_{\{3\}}\right)+\left(\Delta P_{\{1,4\}}-\Delta P_{\{4\}}\right)\right] \\
& +\frac{1 ! \times 2 !}{4 !}\left[\left(\Delta P_{\{1,2,3\}}-\Delta P_{\{2,3\}}\right)+\left(\Delta P_{\{1,2,4\}}-\Delta P_{\{2,4\}}\right)+\left(\Delta P_{\{1,3,4\}}-\Delta P_{\{3,4\}}\right)\right] \\
& +\frac{0 ! \times 3 !}{4 !}\left(\Delta P_{\{1,2,3,4\}}-\Delta P_{\{2,3,4\}}\right) \\
& =33.274 \\
y_{2}^{S p} & =50.623, y_{3}^{S p}=162.454, y_{4}^{S p}=162.454 .
\end{aligned}
$$

That is, the allocation results under Shapley value method are:

$$
x_{1}^{S p}=1133.274, x_{2}^{S p}=256.123, x_{3}^{S p}=2717.934, x_{4}^{S p}=2087.118 .
$$




\subsubsection{MCRS Method Allocation Process}

According to Equations (11)-(12), maximum and minimum allocations of all power stakeholders are:

$$
\begin{aligned}
& x_{1, \max }=1148.961, x_{2, \max }=280.484, x_{3, \max }=2840.34, x_{4, \max }=2209.524, \\
& x_{1, \min }=1100, x_{2, \min }=205.5, x_{3, \min }=2555.48, x_{4, \min }=1924.664
\end{aligned}
$$

According to Equation (13), the sharing proportions of all power stakeholders are:

$$
\gamma_{1} \approx 0.07058, \gamma_{2} \approx 0.10810, \gamma_{3} \approx 0.41066, \gamma_{4} \approx 0.41066
$$

According to Equation (14), allocated synergistic gains of all power stakeholders are:

$$
y_{1}^{M C} \approx 28.855, y_{2}^{M C} \approx 44.191, y_{3}^{M C} \approx 167.879, y_{4}^{M C} \approx 167.88
$$

Hence, the allocation results under MCRS method are:

$$
x_{1}^{M C}=1128.855, x_{2}^{M C}=249.691, x_{3}^{M C}=2723.359, x_{4}^{M C}=2092.544 .
$$

As a result, allocated synergistic gains and allocation results under the nucleolus, Shapley value and MCRS methods are shown in Table 2.

Table 2. Allocated synergistic gains and allocation results (MWh).

\begin{tabular}{ccccccc}
\hline \multirow{2}{*}{ Stakeholder } & \multicolumn{2}{c}{ Nucleolus Method } & \multicolumn{2}{c}{ Shapley Value Method } & \multicolumn{2}{c}{ MCRS Method } \\
\cline { 2 - 7 } & $\begin{array}{c}\text { Allocated Synergistic } \\
\text { Gain }\end{array}$ & $\begin{array}{c}\text { Allocation } \\
\text { Result }\end{array}$ & $\begin{array}{c}\text { Allocated Synergistic } \\
\text { Gain }\end{array}$ & $\begin{array}{c}\text { Allocation } \\
\text { Result }\end{array}$ & $\begin{array}{c}\text { Allocated } \\
\text { Synergistic Gain }\end{array}$ & $\begin{array}{c}\text { Allocation } \\
\text { Result }\end{array}$ \\
\hline 1 & 24.48 & 1124.48 & 33.274 & 1133.274 & 28.855 & 1128.855 \\
2 & 36.587 & 242.087 & 50.623 & 256.123 & 44.191 & 249.691 \\
3 & 173.869 & 2729.349 & 162.454 & 2717.934 & 167.879 & 2723.359 \\
4 & 173.869 & 2098.533 & 162.454 & 2087.118 & 167.88 & 2092.544 \\
\hline
\end{tabular}

The properties of the nucleolus, Shapley value and MCRS methods are compared in terms of existence, uniqueness, rationality and computational feasibility as follows:

- $\quad$ Existence and uniqueness analysis

(1) The nucleolus method satisfies principles of existence and uniqueness if the set of allocated synergistic gains is nonempty, more specifically, if the hybrid generation of wind/solar/hydro power stakeholders could obtain synergistic gains. According to optimal generation models in [14], the existence and uniqueness of nucleolus method can be obtained when the reservoir volume of hydro power stakeholders are abundant;

(2) Since the marginal contribution that a power stakeholder brings to all possible hybrid generation coalitions are available, the Shapley value always exists and could provide a unique allocation result;

(3) As an improvement of nucleolus method, the MCRS method relieves the limitations of nucleolus method by investigating the maximum and minimum allocations of multiple power stakeholders. Therefore, there always exists unique allocation results under MCRS method.

- Rationality analysis

(1) All presented allocation methods satisfy principle of individual rationality, hence the allocation result of a particular power stakeholder is no less than the generation from individual operation, i.e., $x_{i}^{N u} \geq v(i)(i \in N)$, as shown in Table 3; 
(2) All presented allocation methods satisfy principle of coalitional rationality, so that summation of the allocation results of power stakeholders in a particular coalition is no less than the generation from joint operation, i.e., $\sum_{i \in S} x_{i}^{N u} \geq v(S)(S \subseteq N)$, as shown in Table 4;

(3) All presented allocation methods satisfy principle of global rationality, thus the generation of grand coalition $N$ is totally shared by all power stakeholders, i.e., $\sum_{i \in N} x_{i}^{N u}=v(N)$, as shown in Table 5.

Table 3. Individual rationality analysis (MWh).

\begin{tabular}{ccc}
\hline Nucleolus Method & Shapley Value Method & MCRS Method \\
\hline$x_{1}^{N u}=1124.48 \geq v(\{1\})=1100$ & $x_{1}^{S p}=1133.274 \geq v(\{1\})=1100$ & $x_{1}^{M C}=1128.855 \geq v(\{1\})=1100$ \\
$x_{2}^{N u}=242.087 \geq v(\{2\})=205.5$ & $x_{2}^{S p}=256.123 \geq v(\{2\})=205.5$ & $x_{2}^{M C}=249.691 \geq v(\{2\})=205.5$ \\
$x_{3}^{N u}=2729.349 \geq v(\{3\})=2555.48$ & $x_{3}^{S p}=2717.934 \geq v(\{3\})=2555.48$ & $x_{3}^{M C}=2723.359 \geq v(\{3\})=2555.48$ \\
$x_{4}^{N u}=2098.533 \geq v(\{4\})=1924.664$ & $x_{4}^{S p}=2087.118 \geq v(\{4\})=1924.664$ & $x_{4}^{M C}=2092.544 \geq v(\{4\})=1924.664$ \\
\hline
\end{tabular}

Table 4. Coalitional rationality analysis (MWh).

\begin{tabular}{ccc}
\hline Nucleolus Method & Shapley Value Method & MCRS Method \\
\hline$\sum_{i \in\{1,2\}} x_{i}^{N u}=1366.567 \geq v(\{1,2\})=1313.1$ & $\sum_{i \in\{1,2\}} x_{i}^{S p}=1389.397 \geq v(\{1,2\})=1313.1$ & $\sum_{i \in\{1,2\}} x_{i}^{M C}=1378.546 \geq v(\{1,2\})=1313.1$ \\
$\sum_{i \in\{1,3\}} x_{i}^{N u}=3853.829 \geq v(\{1,3\})=3704.441$ & $\sum_{i \in\{1,3\}} x_{i}^{S p}=3851.208 \geq v(\{1,3\})=3704.441$ & $\sum_{i \in\{1,3\}} x_{i}^{M C}=3852.214 \geq v(\{1,3\})=3704.441$ \\
$\sum_{i \in\{1,4\}} x_{i}^{N u}=3223.013 \geq v(\{1,4\})=3073.625$ & $\sum_{i \in\{1,4\}} x_{i}^{S p}=3220.392 \geq v(\{1,4\})=3073.625$ & $\sum_{i \in\{1,4\}} x_{i}^{M C}=3221.399 \geq v(\{1,4\})=3073.625$ \\
$\sum_{i \in\{\{2,3\}} x_{i}^{N u}=2971.436 \geq v(\{2,3\})=2835.964$ & $\sum_{i \in\{2,3\}} x_{i}^{S p}=2974.057 \geq v(\{2,3\})=2835.964$ & $\sum_{i \in\{2,3\}} x_{i}^{M C}=2973.05 \geq v(\{2,3\})=2835.964$ \\
$\sum_{i \in\{2,4\}} x_{i}^{N u}=2340.62 \geq v(\{2,4\})=2205.148$ & $\sum_{i \in\{2,4\}} x_{i}^{S p}=2343.241 \geq v(\{2,4\})=2205.148$ & $\sum_{i \in\{2,4\}} x_{i}^{M C}=2342.235 \geq v(\{2,4\})=2205.148$ \\
$\sum_{i \in\{3,4\}} x_{i}^{N u}=4827.882 \geq v(\{3,4\})=4765.004$ & $\sum_{i \in\{3,4\}} x_{i}^{S p}=4805.052 \geq v(\{3,4\})=4765.004$ & $\sum_{i \in\{3,4\}} x_{i}^{M C}=4815.903 \geq v(\{3,4\})=4765.004$ \\
$\sum_{i \in\{1,2,3\}} x_{i}^{N u}=4095.916 \geq v(\{1,2,3\})=3984.925$ & $\sum_{i \in\{1,2,3\}} x_{i}^{S p}=4107.331 \geq v(\{1,2,3\})=3984.925$ & $\sum_{i \in\{1,2,3\}} x_{i}^{M C}=4101.905 \geq v(\{1,2,3\})=3984.925$ \\
$\sum_{i \in\{1,2,4\}} x_{i}^{N u}=3465.1 \geq v(\{1,2,4\})=3354.109$ & $\sum_{i \in\{1,2,4\}} x_{i}^{S p}=3476.515 \geq v(\{1,2,4\})=3354.109$ & $\sum_{i \in\{1,2,4\}} x_{i}^{M C}=3471.09 \geq v(\{1,2,4\})=3354.109$ \\
$\sum_{i \in\{1,3,4\}} x_{i}^{N u}=5952.362 \geq v(\{1,3,4\})=5913.965$ & $\sum_{i \in\{1,3,4\}} x_{i}^{S p}=5938.326 \geq v(\{1,3,4\})=5913.965$ & $\sum_{i \in\{1,3,3\}} x_{i}^{M C}=5944.758 \geq v(\{1,3,4\})=5913.965$ \\
$\sum_{i \in\{2,3,4\}} x_{i}^{N u}=5069.969 \geq v(\{2,3,4\})=5045.488$ & $\sum_{i \in\{2,3,4\}} x_{i}^{S p}=5061.175 \geq v(\{2,3,4\})=5045.488$ & $\sum_{i \in\{2,3,4\}} x_{i}^{M C}=5065.594 \geq v(\{2,3,4\})=5045.488$ \\
\hline
\end{tabular}

Table 5. Grand rationality analysis (MWh).

\begin{tabular}{cc}
\hline Allocation Method & Global Rationality \\
\hline nucleolus method & $\sum_{i \in\{1,2,3,4\}} x_{i}^{N u}=1124.481+242.572+2729.106+2098.29=v(\{1,2,3,4\})=6194.449$ \\
Shapley value method & $\sum_{i \in\{1,2,3,4\}} x_{i}^{S p}=1133.774+256.623+2717.434+2086.618=v(\{1,2,3,4\})=6194.449$ \\
MCRS method & $\sum_{i \in\{1,2,3,4\}} x_{i}^{M C}=1128.855+249.691+2723.359+2092.544=v(\{1,2,3,4\})=6194.449$ \\
\hline
\end{tabular}

- Computational feasibility analysis

(1) In order to allocate synergistic gains of a n-stakeholder hybrid generation system, the MCRS method needs to solve a sequence of $O(2 n)$ linear programing problems which may bring linear computational burden. However, nucleolus method needs to solve a sequence of $O\left(2^{n}\right)$ linear programing problems and Shapley value method needs to solve a polynomial linear formula with $O\left(2^{n-1}\right)$ terms for each power stakeholder. Hence, both nucleolus and Shapley value method will bring about exponentially growing computational burden.

(2) In a hybrid generation system with large number of wind/solar/hydro power stakeholders, the nucleolus and Shapley value methods may induce combinational explosion problem due to their computational nature, whereas the MCRS method provides a much feasible allocation process by investigating and exploiting the maximum and minimum allocations of all power stakeholders. 
It can be concluded that the nucleolus, Shapley value and MCRS methods could provide positive economic signals to encourage cooperative interactions of multiple power stakeholders. Moreover, both the Shapley value and MCRS methods perform well in terms of existence, uniqueness and rationality, whereas nucleolus method is limited by reservoir volume of hydro power stakeholders. Thus, we consider the Shapley value and MCRS methods as fair, efficient and rational synergistic gains allocation methods for hybrid generation system with multiple wind/solar/hydro power stakeholders. However, according to computational feasibility analysis, Shapley value method may induce combinational explosion problem with the integration of more power stakeholders whereas MCRS method provides a much feasible allocation process.

\subsection{Application of Synergistic Gains Allocation Methods in Yalong River Basin}

A further investigation of the Shapley value and MCRS methods will be applied in a wind-solar-hydro hybrid generation system in Yalong River basin which lies in Southwest China. In this case, there are three wind power stakeholders, two solar power stakeholders and a cascade of four hydro power stakeholders (Jinping I, Jinping II, Guandi and Ertan) as shown in Figure 2. Optimal operations are based on realistic prediction intervals of wind and solar power stakeholders and realistic natural inflow volume of four hydro power stakeholders on 23rd April 2019.

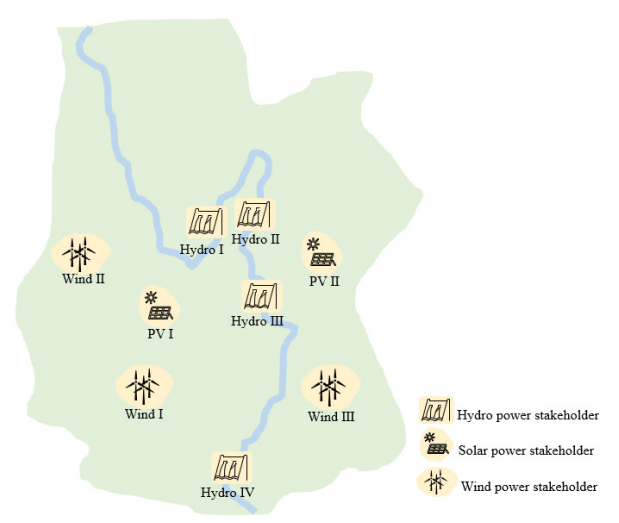

Figure 2. Diagram of wind-solar-hydro hybrid generation system in Yalong River basin.

Next, we will compare the Shapley value and MCRS methods based on the following aspects: - Allocation process

There are totally $511\left(2^{9}-1=511\right)$ possible coalitions for a wind-solar-hydro hybrid generation system with nine power stakeholders.

The Shapley value method requires the information of all 511 hybrid generation coalitions and calculates the weighted marginal contribution that each power stakeholder brings to a total of 256 $\left(C_{8}^{0}+C_{8}^{1}+C_{8}^{2}+C_{8}^{3}+C_{8}^{4}+C_{8}^{5}+C_{8}^{6}+C_{8}^{7}+C_{8}^{8}=256\right)$ hybrid generation coalitions. That is, there are 9 polynomial linear formulas to be solved and each formula consists of 256 terms.

MCRS method requires information of $19(2 \times 9+1=19)$ coalitions and calculates a total of 36 formulas, according to (11)-(14).

It can be indicated that-compared with the Shapley value method-the MCRS method offers a desirable improvement in allocation process for synergistic gains allocation problems with large number of wind/solar/hydro power stakeholders.

- Accuracy of allocation results

Based on allocation results under the Shapley value method, we calculate the error rates of allocation results under the MCRS method as shown in Table 6. 
Table 6. Allocation results and error rates.

\begin{tabular}{cccc}
\hline Power Stakeholder & Shapley Value Method (MWh) & MCRS Method (MWh) & Error Rate (\%) \\
\hline Wind 1 & 711.8629 & 711.9509 & 0.012 \\
Wind 2 & 881.8597 & 881.9519 & 0.010 \\
Wind 3 & 897.7799 & 897.9305 & 0.016 \\
Solar 1 & 415.8892 & 415.9688 & 0.019 \\
Solar 2 & 280.9310 & 280.9804 & 0.018 \\
Hydro 1 & 2520.4546 & 2638.5272 & 4.685 \\
Hydro 2 & 2453.4303 & 2401.5417 & 2.115 \\
Hydro 3 & 1487.2679 & 1536.2185 & 3.291 \\
Hydro 4 & 7127.5545 & 7011.9601 & 1.622 \\
\hline
\end{tabular}

As can be seen from Table 6, the allocation results under the Shapley value method are similar with those under the MCRS method. More specifically, compared with allocation results under Shapley value method, error rates of allocation results under the MCRS method are generally within $4.7 \%$. Therefore, the errors are acceptable and the MCRS method is accurate enough for synergistic gains allocation problems with large number of wind/solar/hydro power stakeholders.

- Computational burden

Computational features of the Shapley value and MCRS methods are compared in the aspects of cost of time and cost of RAM in Table 7.

Table 7. Comparations in computational aspects.

\begin{tabular}{ccc}
\hline Allocation Method & Cost of Time & Cost of RAM \\
\hline Shapley value method & $1750.17 \mathrm{~s}$ & $4.61 \mathrm{MB}$ \\
MCRS method & $21.64 \mathrm{~s}$ & $1.07 \mathrm{MB}$ \\
\hline
\end{tabular}

It is clear that the Shapley value method costs much time and RAM due to its allocation process while the MCRS method dramatically saves time and RAM by investigating and exploiting maximum and minimum allocations of all power stakeholders. Thus, the MCRS method is much practical and computational feasible in allocating problems of a hybrid generation system with large number of wind/solar/hydro power stakeholders.

To conclude, compared with the Shapley value method, the MCRS method significantly simplifies allocating process and improves computational efficiency. Therefore, the MCRS method is recommend as a fair, efficient, rational and computational feasible allocation method for hybrid generation system with large number of wind/solar/hydro power stakeholders.

\section{Conclusions}

Fair, efficient, rational and computational feasible synergistic gains allocation methods are of great importance to encourage hybrid generation of multiple wind/solar/hydro power stakeholders. Based on this principle, cooperative game-based methods (nucleolus, Shapley value and MCRS method) are applied in resolving synergistic gains allocation conflicts among multiple wind/solar/hydro power stakeholders. First, we present elaborate allocation process of the nucleolus, Shapley value and MCRS methods in resolving synergistic gains allocation problems of wind-solar-hydro hybrid generation system. Second, we analyze the presented allocation methods in terms of existence, uniqueness, rationality and computational feasibility. Then, a further application in Yalong River wind-solar-hydro renewable energy basin which lies in southwest China demonstrates that the Shapley value method brings out great computational burden while the MCRS method is much practical and computational feasible. The MCRS method is recommend as a fair, efficient, rational and computational feasible allocation method for hybrid generation system with large number of wind/solar/hydro power stakeholders. 
The probabilistic natural inflow of hydropower will impact the synergistic gains as well as the allocation results. Further work could investigate synergistic gains allocation methods considering the probabilistic natural inflow of hydropower stakeholders.

Author Contributions: All the authors contributed to this work. L.Z. (Liqin Zhang) performed the analysis and simulations. J.X. provided critical guidance to this research and checked the overall logic of this work. X.C. contributed to the conceptual approach on the allocation methods. Y.Z. contributed towards the optimal operations in Yalong River basin. L.Z. (Lv Zhou) contributed towards the allocation of synergistic gains in Yalong River basin. All authors have read and agreed to the published version of the manuscript.

Funding: This research was funded by [the National Natural Science Foundation of China] Grant Number [U1965104] and [U1766203], [the Fundamental Research Funds for the Central Universities of China] Grant Number [2018B01814] and [the National Key R\&D Program of China] Grant Number [2019YFE0105200].

Acknowledgments: This work is supported by the National Natural Science Foundation of China under Grant No. U1965104 and U1766203, the Fundamental Research Funds for the Central Universities of China under Grant No. 2018B01814 and the National Key R\&D Program of China under Grant No. 2019YFE0105200.

Conflicts of Interest: The authors declare no conflict of interest.

\section{Appendix A}

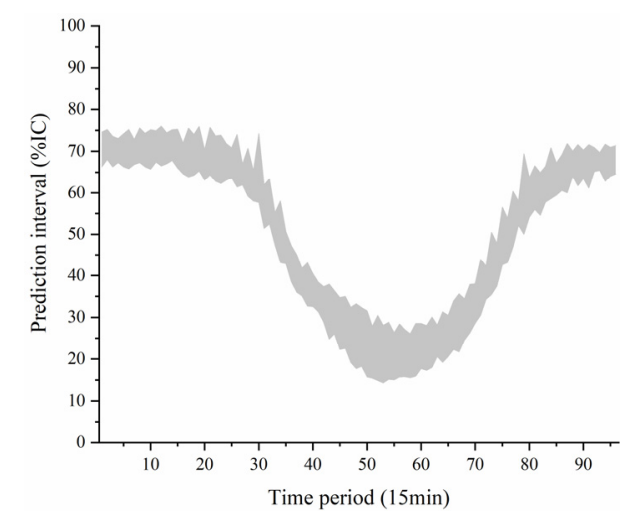

Figure A1. Prediction intervals of wind power stakeholder.

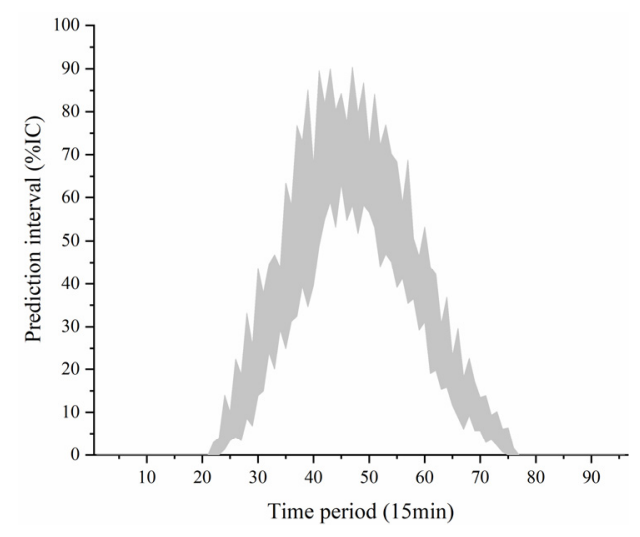

Figure A2. Prediction intervals of solar power stakeholder. 


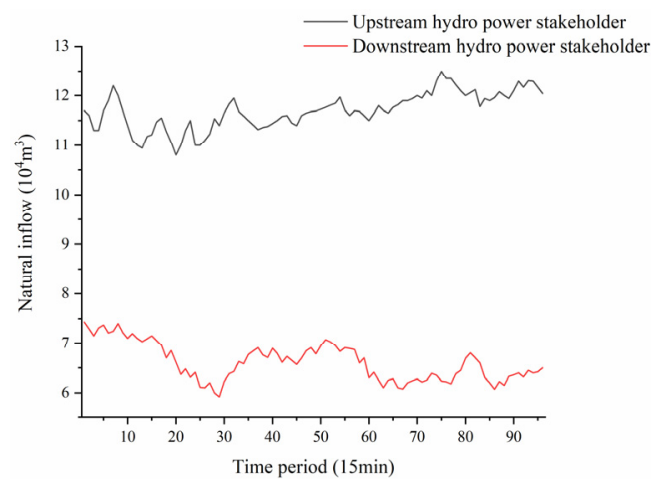

Figure A3. Natural inflow of a cascade of two hydro power stakeholders.

\section{References}

1. Vincenzo, G.D.; Friedler, F.; Huisingh, D. Cleaner energy for sustainable future. J. Clean Prod. 2009, 17, 889-895.

2. Rajput, P.; Malvoni, M.; Kumar, N.M. Operational Performance and Degradation Influenced Life Cycle Environmental-Economic Metrics of mc-Si, a-Si and HIT Photovoltaic Arrays in Hot Semi-arid Climates. Sustainability 2020, 12, 1075. [CrossRef]

3. Appavou, F.; Brown, A.; Epp, B.; Gibb, D.; Kondev, B.; McCrone, A.; Murdock, H.E.; Musolino, E.; Ranalder, L.; Sawin, J.L.; et al. Renewables 2019- Global Status Report; REN21: Paris, France, 2019.

4. Zhang, X.; Ma, G.; Huang, W.; Chen, S.; Zhang, S. Short-Term Optimal Operation of a Wind-PV-Hydro Complementary Installation: Yalong River, Sichuan Province, China. Energies 2018, 11, 868. [CrossRef]

5. Wei, H.; Hong, X.Z.; Yu, D. Short-term optimal operation of hydro-wind-solar hybrid system with improved generative adversarial networks. Appl. Energy 2019, 250, 389-403. [CrossRef]

6. Li, J.; Wang, S.; Ye, L. A coordinated dispatch method with pumped-storage and battery-storage for compensating the variation of wind power. Prot. Control Mod. Power Syst. 2018, 3, 21-34. [CrossRef]

7. Almeida, V.; Getschko, D.; Afonso, C. The Origin and Evolution of Multistakeholder Models. IEEE Internet Comput. 2015, 19, 74-79. [CrossRef]

8. Faria, E.; Barroso, L.A.; Kelman, R. Allocation of Firm-Energy Rights Among Hydro Plants: An Aumann-Shapley Approach. IEEE Trans. Power Syst. 2009, 24, 541-551. [CrossRef]

9. Faria, V.; de Queiroz, A.; Lima, L. Cooperative game theory and last addition method in the allocation of firm energy rights. Appl. Energy 2018, 226, 905-915. [CrossRef]

10. Zhang, X.; Liu, P.; Xu, C.Y. Derivation of Hydropower Rules for Multireservoir Systems and Its Application for Optimal Reservoir Storage Allocation. J. Water Resour. Plan. Manag. 2019, 145, 04019010. [CrossRef]

11. Yang, H.; Yu, Q.; Liu, J. Optimal Wind-Solar Capacity Allocation with Coordination of Dynamic Regulation of Hydropower and Energy Intensive Controllable Load. IEEE Access 2020, 8, 110129-110139. [CrossRef]

12. Zhang, X.; Lv, J.; Xie, J.; Yu, J. Solar Radiation Allocation and Spatial Distribution in Chinese Solar Greenhouses: Model Development and Application. Energies 2020, 13, 1108. [CrossRef]

13. Xu, B.; Ma, Y.; Zhong, P.A. Bargaining Model of Synergistic Revenue Allocation for the Joint Operations of a Multi-Stakeholder Cascade Reservoir System. Water Resour. Manag. 2018, 32, 4625-4642. [CrossRef]

14. Xie, J.; Zhang, L.Q.; Chen, X.Y. Incremental Benefit Allocation for Joint Operation of Multi-stakeholder Wind-PV-Hydro Complementary Generation System with Cascade Hydro-power: An Aumann-Shapley value method. IEEE Access 2020, 8, 68668-68681. [CrossRef]

15. Gan, D.Q.; Feng, D.H.; Xie, J. Electricity Markets and Power System Economics; CRC, Taylor \& Francis: Boca Raton, FL, USA, 2013.

16. Baeyens, E.; Bitar, E.Y.; Khargonekar, P.P. Coalitional Aggregation of Wind Power. IEEE Trans. Power Syst. 2013, 28, 3774-3784. [CrossRef]

17. Tzavellas, A.; Nguyen, P.; Ribeiro, P. A game theory approach for coordinating multiple virtual synchronous generators. In Proceedings of the 2013 IEEE Grenoble Conference, Grenoble, France, 16-20 June 2013. 
18. Alexandre, S.; Lima, D.A.; Freire, L.; Contreras, J. Sharing quotas of a renewable energy hedge pool: A cooperative game theory approach. In Proceedings of the 2011 IEEE Trondheim PowerTech, Trondheim, Norway, 19-23 June 2011.

19. Zima-Bockarjova, M.; Matevosyan, J.; Zima, M. Sharing of Profit from Coordinated Operation Planning and Bidding of Hydro and Wind Power. IEEE Trans. Power Syst. 2010, 25, 1663-1673. [CrossRef]

20. Heaney, J.P.; Dickinson, R.E. Methods for apportioning the cost of a water resource project. Water Resour. Res. 1982, 18, 476-482. [CrossRef]

(C) 2020 by the authors. Licensee MDPI, Basel, Switzerland. This article is an open access article distributed under the terms and conditions of the Creative Commons Attribution (CC BY) license (http://creativecommons.org/licenses/by/4.0/). 